\title{
Relación esquelética según Pérez y Kim y proyección USP en pacientes adultos ortodóncicos
}

\author{
Skeletal relationship according to Pérez, Kim and USP projection in orthodontic \\ adult patients
}

\author{
Yury Miguel Tenorio Cahuana ${ }^{1 \mathrm{a}}$ \\ ${ }^{1}$ Escuela Profesional de Odontologia de la Universidad Nacional Jorge Basadre Grohmann. Tacna, Perú. \\ ${ }^{a}$ Docente, Especialista en Ortodoncia y Ortopedia Maxilar.
}

\begin{abstract}
Resumen
Objetivo: Determinar la relación esquelética sagital utilizando los cefalogramas de Perez, Kim y Proyección USP para luego determinar la concordancia entre dichos estudios cefalométricos, teniendo en cuenta la longitud e inclinación de la base craneal anterior en pacientes adultos ortodóncicos. Metodología: Estudio de tipo correlacional, retrospectivo y de corte transversal. La población estuvo constituida por 135 pacientes adultos atendidos en tres consultorios especializados de la ciudad de Tacna en el año 2017. Conclusiones: 1) Existe muy buena concordancia entre los cefalogramas de Kim y proyección USP con 0.913 , los cefalogramas de Kim y proyección USP con 0.827 , así como buena concordancia entre los cefalogramas de Pérez y Kim con 0.746 , considerando los parámetros de la longitud e inclinación de la base craneal anterior. 2) Se encontró buena concordancia entre los cefalogramas de Pérez y Proyección USP con 0.698 y moderada concordancia entre los cefalogramas de Pérez y Kim con 0.549 y cefalogramas de Kim y Proyección USP con 0.591 , sin tomar en cuenta los parámetros de la longitud e inclinación de la base craneal anterior.

Palabras clave: Relación esquelética, base craneal anterior, cefalograma.
\end{abstract}

\begin{abstract}
Objective: To determine the sagittal skeletal relationship using Perez and Kim's cephalograms and USP Projection and then determine the concordance between those cephalometric studies, taking into account the length and inclination of the anterior cranial base in adult orthodontic patients. Methodology: Correlational, retrospective and cross-sectional study. The population consisted of 135 adult patients treated in three specialized offices in the city of Tacna in 2017. Conclusions: 1) There is very good agreement between Kim's cephalograms and USP projection with 0.913 , Kim's cephalograms and USP projection with 0.827 , as well as good concordance between Perez and Kim's cephalograms with 0.746 , considering the parameters of the length and inclination of the anterior cranial base. 2) Good concordance was found between Pérez and Projection USP with 0.698 and moderate concordance between Pérez and Kim's cephalograms with 0.549 and Kim and USP Projection cephalograms with 0.591, without taking into account the parameters of the of the anterior cranial base.
\end{abstract}

Keywords: Skeletal relationship, anterior cranial base, cephalogram. 


\section{Introducción}

La relación esquelética es primordial para fundamentar el diagnóstico, evaluar un plan de tratamiento para tratar las diferentes maloclusiones en la especialidad de ortodoncia. El clínico utiliza diversos análisis cefalométricos para determinar la relación esquelética desde el perfil de la cefalometría; asimismo, muchos de ellos utilizan la base craneal anterior conjuntamente con las bases óseas del maxilar y la mandíbula para clasificar las relaciones esqueléticas. Sin embargo, la base craneal anterior presenta muchas variaciones geométricas, especialmente el punto Nasion, lo cual nos da como resultado clasificaciones que resultan inexactas $(1,11,21-23)$.

La evaluación de la relación sagital entre la maxila y la mandíbula es muy compleja por el número de estructuras óseas que los acompañan por ello es importante reconocer sus vectores de crecimiento. La cefalometría se preocupa en relacionar en el sentido anteroposterior dichos maxilares para poder cuantificar su grado de discrepancia ósea $(2,12-14)$.

Hoy en día los clínicos utilizan varios análisis cefalométricos dentro de los exámenes auxiliares para poder diagnosticar correctamente y clasificar la relación esquelética, pero muchos de los casos no concluyen en el mismo diagnóstico $(3,4)$.

El análisis de Pérez (5) utilizó los parámetros de Steiner para poder determinar la relación cefalométrica sagital con el tipo de crecimiento en la población mexicana. Para alcanzar tales fines, el autor utilizó los parámetros de Steiner, base craneal anterior (Silla a Nasion) además de los puntos A en la maxila y el punto $\mathrm{B}$ en la mandíbula; además de realizar un aporte personal empleando las alturas faciales anterior $y$ posterior del análisis de Jarabak. En este estudio se determinó diversas desviaciones estándar de las relaciones esqueléticas clase I, II y III.

El análisis de Kim (6) se vale del indicador de la displasia antero posterior (APDI) para lo cual utilizó el plano facial (Nasion a Pogonion) puntos A y B, el plano palatino (Espina Nasal Anterior a Espina Nasal Posterior) y el plano de Frankfort (Porion a Orbitario) a través del cual se puede diferenciar el patrón anteroposterior de la maloclusión.

El análisis de la Proyección USP emplea el plano ENP - P' (Espina Nasal Posterior a P' que vendría a ser la proyección de Nasion en el plano palatino) y el plano mandibular (Gonion cefalométrico a Mentoniano). Este análisis fue propuesto por los profesores Sebastian Interlandi y Augusto Sato de la Universidad de Sao Paulo. El análisis relaciona la maxila y mandíbula a través de una bisectriz formada por los planos antes mencionados y la proyección de los puntos Ay B sobre esta (1,7-10).

\section{Material y métodos}

El tipo de investigación fue descriptivo, de tipo retrospectivo, de acuerdo al registro de información y ocurrencia de los hechos; $y$ transversal de acuerdo al período y ocurrencia de los hechos.

La población estuvo constituida por 135 pacientes atendidos en tres consultorios especializados en el año 2017. Los criterios de inclusión fueron pacientes adultos sin tratamientos de previos de ortodoncia que no presentaban ningún síndrome o asimetría y cuyas radiografías laterales de cráneo estuvieran en condiciones óptimas para realizar trazados.

La muestra del estudio de investigación se obtuvo con el método probabilístico aleatorio simple, con la fórmula estadística para poblaciones infinitas de variable cualitativa, con un grado de confianza del $95 \%$ (1.96) y con la probabilidad de error de $5 \%$; lo que resultó en 100 unidades de estudio.

Se realizaron tres análisis cefalométricos por cada radiografía cefalométrica. Los trazos de las estructuras anatomo-radiológicas fueron realizadas en papel acetato (hojas cefalométricas). Sobre cada teleradiografía se trazaron estructuras anátomoradiológicas y se ubicaron los puntos cefalométricos de interés para demarcar la altura facial anterior y altura facial posterior y determinar el tipo de crecimiento. También, se hallaron los puntos cefalométricos Porion - Orbitario, Silla y Nasion para encontrar los planos de Frankfort y base craneal anterior respectivamente (1518,26-29).

El ángulo ANB de Steiner se desde el punto $\mathrm{A}$ (maxilar superior), punto $\mathrm{N}$ (Nasion) y el punto $\mathrm{B}$ (mandíbula). El Indicador de displasia antero posterior se realizan los trazados de los planos de Frankfort, plano facial, plano palatino, plano AB. Finalmente, se traza la proyección USP que son las proyecciones ortogonales de los puntos A y B sobre el plano de la bisectriz del ángulo formado por el plano ENP-P' y el plano Go-Me; todos estos cefalogramas determinan la relación esquelética sagital $(5,6,27)$.

Para el cálculo del error en la obtención de las medidas cefalométricas, se seleccionó de manera aleatoria 20 placas. Después de 90 días, las placas fueron trazadas nuevamente por el mismo operador y se obtuvo 60 medidas cefalométricas, las mismas que fueron comparadas con las medidas originales a través de la prueba de Dahlberg.

La recolección de la información del presente estudio, se utilizó una ficha de recolección de datos en base a las dimensiones e indicadores de la variable en estudio, el cual fue validado por el juicio de tres expertos.

La información obtenida se procesó y codificó en la base de datos elaborado en el programa excel 
2010, posteriormente, se procesó con el paquete estadístico SPSS y se presentó en tablas de frecuencia y cruzadas.

\section{Resultados}

En la Tabla 1 se muestra que de 100 cefalogramas solo 19 cumplieron los parámetros de la longitud e inclinación de la base craneal anterior.

Tabla 1. Frecuencia y porcentaje de la longitud e
inclinacion de la base craneal anterior
dentro y fuera de los parametros

En la Tabla 2 se aprecia que el total de cada una de las columnas (por cada sistema) es igual a 100. Como se puede apreciar, las 3 distribuciones son marcadamente diferentes. En los cefalogramas de Pérez y Proyección USP, la mayor parte de casos se ubican en la categoría clase II mientras que Kim la mayor parte es en la categoría clase I.

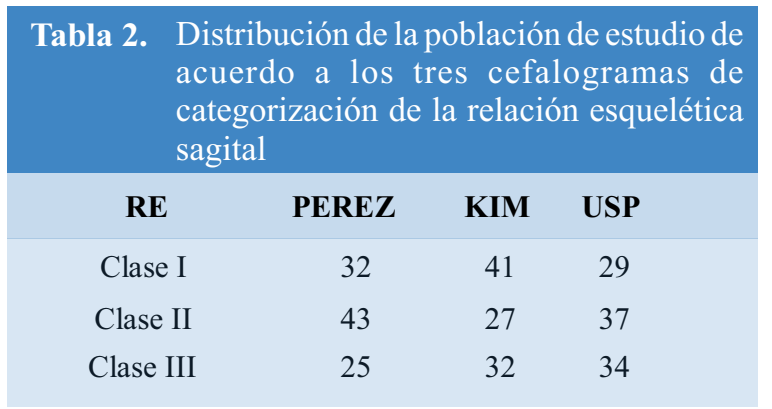

En la Tabla 3 se puede apreciar la concordancia entre los cefalogramas de Pérez y Kim, considerando los parámetros de la longitud e inclinación de la base craneal anterior $16(2+5+9)$.

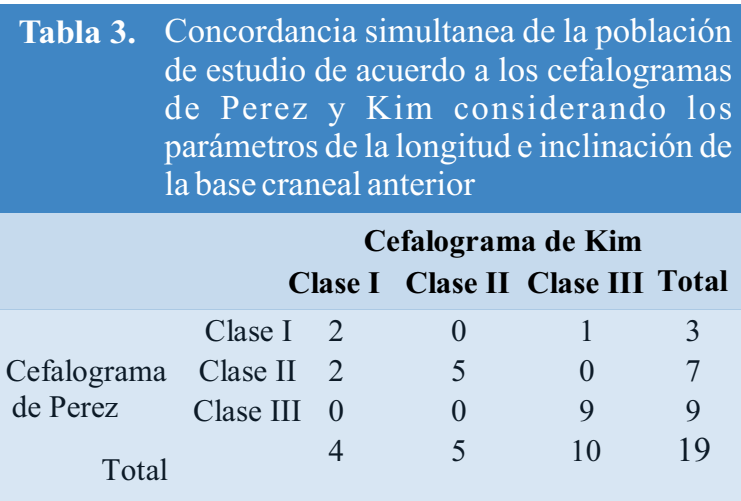

\begin{tabular}{|c|c|c|c|c|c|}
\hline Tabla 4. & & las & ase & ase & \\
\hline \multirow{4}{*}{$\begin{array}{l}\text { Cefalograma } \\
\text { de Kim }\end{array}$} & Clase I & 3 & 1 & 0 & 4 \\
\hline & Clase II & 0 & 5 & 0 & 5 \\
\hline & Clase III & 0 & 0 & 10 & 10 \\
\hline & & 3 & 6 & 10 & 19 \\
\hline
\end{tabular}

En la Tabla 5 se observa que la concordancia entre los cefalogramas de Pérez y USP, considerando los parámetros de la longitud e inclinación de la base craneal anterior, es $17(2+6+9)$

\begin{tabular}{|c|c|c|c|c|c|}
\hline \multirow[t]{2}{*}{$\begin{array}{l}\mathrm{C} \\
\mathrm{d} \\
\mathrm{d} \\
\mathrm{p} \\
\mathrm{p} \\
\text { la }\end{array}$} & \multicolumn{5}{|c|}{$\begin{array}{l}\text { Concordancia simultanea de la población } \\
\text { de estudio de acuerdo a los cefalogramas } \\
\text { de Perez y USP, considerando los } \\
\text { parametros de la longitud e inclinacion de } \\
\text { la base craneal anterior }\end{array}$} \\
\hline & & Clase & & $\begin{array}{l}\text { de US } \\
\text { ase III }\end{array}$ & Total \\
\hline \multirow{3}{*}{$\begin{array}{l}\text { Cefalograma } \\
\text { de Perez }\end{array}$} & Clase I & 2 & 0 & 1 & 3 \\
\hline & Clase II & 1 & 6 & 0 & 7 \\
\hline & Clase III & 0 & 0 & 9 & 9 \\
\hline Total & & 3 & 6 & 10 & 19 \\
\hline
\end{tabular}

En la Tabla 6 se observa que el valor de Kappa es de 0.746 , lo cual significa que la concordancia entre los cefalogramas de Pérez y Kim -considerando los parámetros de la longitud e inclinación de la base craneal anterior- es catalogada como buena.

\begin{tabular}{|c|c|c|c|c|}
\hline Tabla 6 & \multicolumn{4}{|c|}{$\begin{array}{l}\text { Concordancia simultanea de la población } \\
\text { de estudio de acuerdo a los cefalogramas } \\
\text { de Perez y USP, considerando los } \\
\text { parametros de la longitud e inclinacion de } \\
\text { la base craneal anterior }\end{array}$} \\
\hline \multicolumn{5}{|c|}{$\begin{array}{l}\text { Error } \\
\text { Valor estándar Aprox. S }{ }^{\mathrm{b}} \text { Aprox. Sig. } \\
\text { Asintótico }\end{array}$} \\
\hline $\begin{array}{l}\text { Medida } \\
\text { de acuerdo }\end{array}$ & Карра .746 & 126 & 4.501 & .000 \\
\hline $\mathrm{n}^{\mathrm{o}}$ de casos & válidos 19 & & & \\
\hline
\end{tabular}

a. No se presupone la hipótesis nula.

b. Utilización del error estándar asintótico que presupone la hipótesis nula.

En la Tabla 7 se observa que el valor de Kappa es de 0.913 , lo cual significa que la concordancia entre los cefalogramas de Kim y Proyección USP - 
considerando los parámetros de la longitud e inclinación de la base craneal anterior- es catalogada como muy buena.

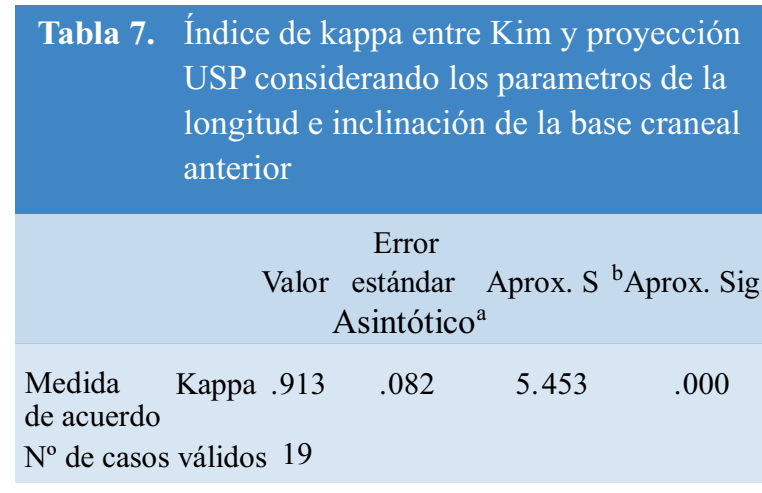

a. No se presupone la hipótesis nula.

b. Utilización del error estándar asintótico que presupone la hipótesis nula.

En la Tabla 8 se observa que el valor de Kappa es de 0.827 , lo cual significa que la concordancia entre los cefalogramas de Pérez y Proyección USP considerando los parámetros de la longitud e inclinación de la base craneal anterior- es catalogada como muy buena.

Tabla 8. Índice de Kappa entre Perez y proyección
USP considerando los parametros de la
longitud e inclinacion de la base craneal
anterior
Valor $\begin{gathered}\text { Estándar Aprox. } \mathrm{S}^{\mathrm{b}} \text { Aprox. Sig. } \\ \text { Asintótico a }\end{gathered}$
$\begin{gathered}\text { Medidade } \\ \text { acuerdo } \\ \mathrm{N}^{\mathrm{o}} \text { de casos válidos } 19\end{gathered}$

a. No se presupone la hipótesis nula.

b. Utilización del error estándar asintótico que presupone la hipótesis nula.

\section{Discusión}

En la Tabla 1 se demuestra la frecuencia y porcentaje dentro y fuera de los parámetros de la longitud e inclinación de la base craneal anterior, de ellos solamente 19 cefalogramas cumplían con tales parámetros y 81 cefalogramas no. Estos resultados concuerdan con el estudio de Tenorio (19).

En la tabla 2 se aprecia la distribución de la población de estudio de acuerdo a los 3 cefalogramas de categorización de la relación esquelética sagital $(\mathrm{N}=$ 100). Los datos nos indican que la mayor parte de casos se ubica en la categoría clase II en los cefalogramas de Pérez y Proyección USP y la categoría Clase I en Kim. Esos resultados concuerdan con el estudio de Acuña (15) con la diferencia de los cefalogramas de Pérez y Steiner.

La tabla 3 relaciona los cefalogramas de Pérez y Kim tomando en cuenta los parámetros de la longitud e inclinación de la base craneal anterior. De acuerdo a los datos, la coincidencia total fue de $16 \%$, en donde la mayor coincidencia fue dada para los de la clase III con un $9 \%$; por otro lado, la menor fue dada para los de la clase II con un $5 \%$ y para los de la clase I con un $2 \%$.

La tabla 4 relaciona los cefalogramas de Kim y USP tomando en cuenta los parámetros de la longitud e inclinación de la base craneal anterior. Aquí, la coincidencia total fue de $18 \%$, donde la mayor coincidencia fue dada para los de la clase III con un 10 $\%$; por otro lado, la menor fue dada para los de la clase II con un $5 \%$ y para los de la clase I con un $3 \%$. Estos resultados concuerdan con el estudio de Luna (20); sin embargo, en este estudio no se toma en cuenta los parámetros de la longitud e inclinación de la base craneal anterior.

Analizando las coincidencias por pares de la Tabla 5 y considerando los parámetros de la longitud e inclinación de la base craneal anterior entre los cefalogramas de Pérez y Proyección USP, se determinó una coincidencia total de $17 \%$ según las siguientes proporciones: clase III en $9 \%$, clase II en $6 \%$ y clase I en $2 \%$.

En las tablas 6, 7 y 8, al aplicar la prueba estadística índice de Kappa tomando en cuenta los parámetros de la longitud e inclinación de la base craneal anterior, se encontró una buena concordancia entre los cefalogramas de Pérez y Kim con 0.746, así como muy buena concordancia entre los cefalogramas de Kim y Proyección USP con 0.913 y los cefalogramas de Pérez y Proyección USP con 0.827. Estos resultados difieren con el trabajo de investigación de Tenorio (30) en la cual la concordancia entre los cefalogramas de Steiner y Kim con 0.136 es pobre y los cefalogramas de Steiner y Proyección USP con 0.205 es baja.

\section{Conclusiones}

1. Existe muy buena concordancia entre los cefalogramas de Kim y Proyección USP con 0.913 y los cefalogramas de Kim y Proyección USP con 0.827 , buena concordancia entre los cefalogramas de Pérez y Kim con 0.746, tomando en cuenta los parámetros de la longitud e inclinación de la base craneal anterior.

2. Se encontró buena concordancia entre los cefalogramas de Pérez y proyección USP con 
0.698 y moderada concordancia entre los cefalogramas de Pérez y Kim con 0.549 y cefalogramas de Kim y Proyección USP con 0.591 , sin tomar en cuenta los parámetros de la longitud e inclinación de la base craneal anterior.

3. Las 3 distribuciones son marcadamente diferentes: en los cefalogramas de Pérez y Proyección USP, la mayor parte de casos se ubica en la categoría clase II; mientras que en Kim, la mayor parte está en la categoría clase I.

\section{Recomendaciones}

1. Se recomienda tener en cuenta, en primer lugar, la longitud e inclinación de la base craneal anterior

\section{Referencias bibliográficas}

1. Interlandi S, Ortodoncia bases para la iniciación. Editorial Artes médicas latinoamericana. Sao Paulo. 1998. p. 440

2. Quiroz O. La Base Craneal Anterior, consideraciones en su longitud e inclinación. Acta odontológica Venezolana. Universidad Central de Venezuela. 2002.p. 2

3. Aguila J. Manual de Cefalometría. Ed. Actualidades Médico Odontológicas Latinoamericana. Caracas. 1966.p. 49

4. Aguila J. Tratado de Ortodoncia. Editorial Actualidades Médico Odontológicas Latinoamericana. Caracas; 2000.pp. 34-47

5. Pérez C. Tratado de cefalometría; un análisis sencillo, lógico y preciso para ortodoncia y ortopedia dentofacial. Amolca. Venezuela;2013.p. 49

6. Kim Y.H, Vietas, J.J. Anteroposterior dysplasia indicator: an adjunct to cephalometric differential diagnosis. American Journal Orthodontics. 73(6):61933,June. 1978.

7. Acuña E. Estudio comparativo del Cefalograma de Kim, Steiner y proyección USP en la determinación de la Relación Esquelética Sagital. UNMSM. Lima - Perú; 2011.p. 33

8. Ram S, Nanda R, Merril R. Cephalometric assessment of sagital relationship between maxilla and mandible. American Journal Orthodontics Dentofacial Orthopedic. 15(4):328-44,Apr. 1994.

9. Lux C, Burden D, Conradt C, Komposch G. Age- para determinar una relación esquelética sagital posicional, aplicar los tres cefalogramas en conjunto.

3. Realizar más estudios del cefalograma de Pérez en nuestro medio específicamente en pacientes con crecimiento.

4. En la aplicación del cefalograma de Kim, se debe tener en cuenta la identificación y el trazado de los puntos cefalométricos de referencia Porion y espina nasal anterior debido a su variabilidad.

5. Utilizar el cefalograma de Proyección USP como alternativa en el caso de que las referencias anatómicas no sean visibles.

6. Estudiar otros análisis cefalométricos como el ángulo Beta, Wits modificado, la línea Yen, ángulo bisector de Frankfort que se pueden aplicar en nuestro medio.

related changes in sagittal relationship betweeen the maxilla and mandible. European Journal of Orthodontics. 27(6):568-78,Dec. 2005.

10. Al - Hammadi A, Labib A, Heider A, Sultan M. Overjet dentoesquelético: un nuevo método para evaluar la relación máxilo-mandibular sagital. Australian Journal of Basic and Applied Sciences, 5(9): 1830-1836. 2011

11. Zamora A, Medellin R, Elorza-Pérez H. Relación de la deflexión craneal y longitud de la base craneal anterior. Ortodoncia actual, 2019; 8(29).

http://www.imbiomed.com/1/1/articulos.php?id_revist $\mathrm{a}=326 \&$ id_ejemplar $=8064$

12. Giraldo A, Arbelaez R, Gomez J. Caracterización cefalométrica de la relación sagital de los maxilares en escolares entre los 5 y 6 años de edad. Revista colombiana de investigación en odontología, 2012; 3 ( 8$)$

https://www.rcio.org/index.php/rcio/article/view/93/2 02

13. Neela PK, Mascarenhas R, Husain A. A new sagittal displasia indicator: The Yen angle. World Journal orthodontics. 10(2):147-51.

14. Marengo H, Romani N. Estudio cefalométrico comparativo para el diagnóstico anteroposterior de las bases apicales entre los ángulos ANB y la Proyección USP. Revista Científica Odontológica Sanmarquina. 9(2): 8-11. 2006

15. Acuña EC. Estudio comparativo del cefalograma de Kim, Steiner y proyección USP en la determinación de la relación esquelética sagital. Tesis para optar título de cirujano dentista. Facultad de Odontología Universidad Nacional Mayor de San Marcos. Lima - 
Perú. 2011

16. Pachas LV. Evaluación de la relación sagital de los maxilares de una población peruana de niños comprendidos entre 7 y 12 años de edad, según el análisis cefalométrico de la proyección USP. Tesis para optar título de cirujano dentista. Facultad de Odontología - Universidad Nacional Mayor de San Marcos. Lima-Perú. 2014

17. Pino E. Evaluación cefalométrica de la inclinación y longitud de la base craneal anterior en pacientes entre 8 y 12 años atendidos en el servicio de odontopediatría de la clínica de la UPCH entre los años 2010 - 2016. Tesis para optar título de cirujano dentista. Facultad de Odontología - Universidad Particular Cayetano Heredia. Lima-Perú. 2017

18. Flores A. Evaluación cefalométrica de la longitud e inclinación de la base craneal anterior en pacientes con diferente relación esquelética. Tesis para optar título de cirujano dentista. Facultad de Odontología Universidad Nacional Mayor de San Marcos. Lima Perú. 2017

19. Tenorio YM. Relaciones de la Base Craneal Anterior en su longitud e inclinación - Tacna. Revista Médica Basadrina - Universidad Nacional Jorge Basadre Grohmann, 2012; 8(1). pp 21-23. Ene-Mar. 2014.

20. Luna S. Patrón esqueletal sagital mediante la comparación de los cefalogramas de Steiner, Ricketts y Proyección USP con fines de diagnóstico ortodóncico de pacientes niños que asistieron a la clínica odontológica de la UNJBG en el periodo 2012 al 2015. Tesis para optar título de cirujano dentista. Facultad de Odontología - Universidad Nacional Mayor de San Marcos. Lima-Perú. 2016

21. Aguila J, Enlow D. Crecimiento Craneofacial. Ortodoncia y Ortopedia. Editorial Actualidades Médico Odontológicas Latinoamericana. Caracas. 1991.p. 2
22. Infante CL. Fundamentos para la evaluación del crecimiento, desarrollo y función craneofacial. Universidad Nacional de Colombia, Bogotá (2009). I S B N 9789584442864 . P p . 15-21. http://bdigital.unal.edu.co/634.

23. Fernandez J, Gabriel Da Silva O. Atlas cefalometría y análisis facial. Centro de estudios de ortodoncia del Bajío. Editorial Médica. Ripano. Madrid (2009). pp. 13, 29

24. Vellini FF. Ortodoncia: Diagnóstico y Planificación clínica. Editorial Artes Médicas, 1a Ed. 2002. Pp. 314 327.

25. Racosi T, Jonas I. Atlas de Ortopedia Maxilar, Diagnóstico. Editorial Masson - Salvat. Alemania 1996. p.184

26. Quiroz O. Manual de Ortopedia Funcional de los Maxilares y Ortodoncia Interceptiva. Actualidades médico odontológicas latinoamérica, C.A. 2000. Páginas 20 - 21

27. Silva R. Young H. Kim cephalometric analytic procedure. Latinoamerican University, ULA. México D.F

28. Bimler HP. Los modeladores elásticos y análisis cefalométrico compacto. Editorial Actualidades Médico Odontológicas Latinoamericana, CA, primera edición 1993. Caracas - Venezuela. Página 129

29. Rodriguez MD, Janson GRP, De Almeida RR, Pinzan A, Henriques JFC y De Freidas MR. Atlas de

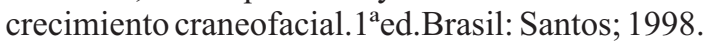

30. Tenorio YM. Comparación de los cefalogramas de Kim, Steiner y USP en la determinación de la relación esqueletal sagital, tomando en cuenta la similitud de los parámetros de la inclinación y la longitud de la base craneal anterior - Tacna 2014. Universidad Nacional Jorge Basadre Grohmann. R.F. N ${ }^{\circ} 14174-2014-$ FACS-UNJBG
Correspondencia:

ymtc@yahoo.com
Fecha de recepción : 25 de junio de 2019

Fecha de aceptación : 08 de octubre de 2019 